\title{
Graphene-Semiconductor Catalytic Nanodiodes for Quantitative Detection of Hot Electrons Induced by a Chemical Reaction
}

\author{
Hyosun Lee ${ }^{1,2}$, Ievgen I. Nedrygailov ${ }^{1,2}$, Young Keun Lee $e^{1,2}$, Changhwan Lee $e^{1,2}$, Hongkyw \\ $\mathrm{Choi}^{3}$, Jin Sik $\mathrm{Choi}^{3}$, Choon-Gi Choi ${ }^{3}$, and Jeong Young Park ${ }^{1,2 *}$ \\ ${ }^{1}$ Graduate School of EEWS, Korea Advanced Institute of Science and Technology (KAIST), \\ Daejeon 305-701, Republic of Korea \\ ${ }^{2}$ Center for Nanomaterials and Chemical Reactions, Institute for Basic Science (IBS), \\ Daejeon 305-701, Republic of Korea \\ ${ }^{3}$ Creative Research Center for Graphene Electronics, Electronics and Telecommunications \\ Research Institute (ETRI),Daejeon 305-700, Republic of Korea
}

*To whom correspondence should be addressed. E-mail: jeongypark@kaist.ac.kr 


\section{- Growth and transfer of graphene}

Single graphene sheets were grown using ambient-pressure chemical vapor deposition (CVD) on pre-annealed $\mathrm{Cu}$ foil (Alta Aesar, 99.9999\% purity, 25 um thick). The synthesis was performed at $1000{ }^{\circ} \mathrm{C}$ with a gas mixture of $\mathrm{CH}_{4}(30 \mathrm{sccm})$ and $\mathrm{H}_{2}(10 \mathrm{sccm})$ under ambient pressure. To form a monolayer of graphene, the chamber was cooled rapidly (cooling rate: $\left.\cong 32{ }^{\circ} \mathrm{C} / \mathrm{min}\right)$ under $\operatorname{Ar}(100 \mathrm{sccm})$ after graphene growth. Once the chamber cooled to room temperature, the $\mathrm{Cu}$ foil was removed from the furnace. To transfer the asgrown graphene on $\mathrm{Cu}$ foil to the prepared device, poly(methyl methacrylate) (PMMA) was spun over one side of the graphene/Cu substrate. Next, the backside of the $\mathrm{Cu}$ foil and graphene (i.e., where no PMMA was deposited) was etched using a $0.1 \mathrm{M}$ ammonium persulfate solution, releasing the PMMA/graphene bi-layer. This PMMA/graphene film was transferred from the solution onto the selected area of the device after rinsing multiple times. Finally, the PMMA on top of the graphene was removed using a wet chemical treatment in acetone and baked for better quality. After transfer, the presence and quality of the singlelayer graphene were confirmed using Raman spectroscopy, X-ray photoelectron spectroscopy (XPS), and scanning electron microscopy (SEM) (Figures S1, S2, and S3, respectively). Thermal and chemical stability of the single graphene layer under reaction conditions (i.e., high temperature and $\mathrm{H}_{2}$ oxidation atmosphere) were also checked by measuring the Raman spectrum before and after $\mathrm{H}_{2}$ oxidation (see Figure S1). Raman spectra were obtained at ambient conditions using a LabRAM HR UV-VIS-NIR Raman microscope (Horiba Jobin Yvon). A laser source with an excitation energy of $2.41 \mathrm{eV}\left(\lambda_{D}=514.5 \mathrm{~nm}\right)$ was used. XPS spectra were acquired by using a Thermo VG Scientific Sigma Probe spectrometer equipped with $\mathrm{n}$ Al-K $\alpha$ X-ray source $(1486.3 \mathrm{eV})$ with an energy resolution of $0.5 \mathrm{eV}$ full width at half maximum under ultra-high vacuum conditions of $10^{-10}$ Torr. Field emission SEM was conducted using a Magellan 400 SEM instrument. 


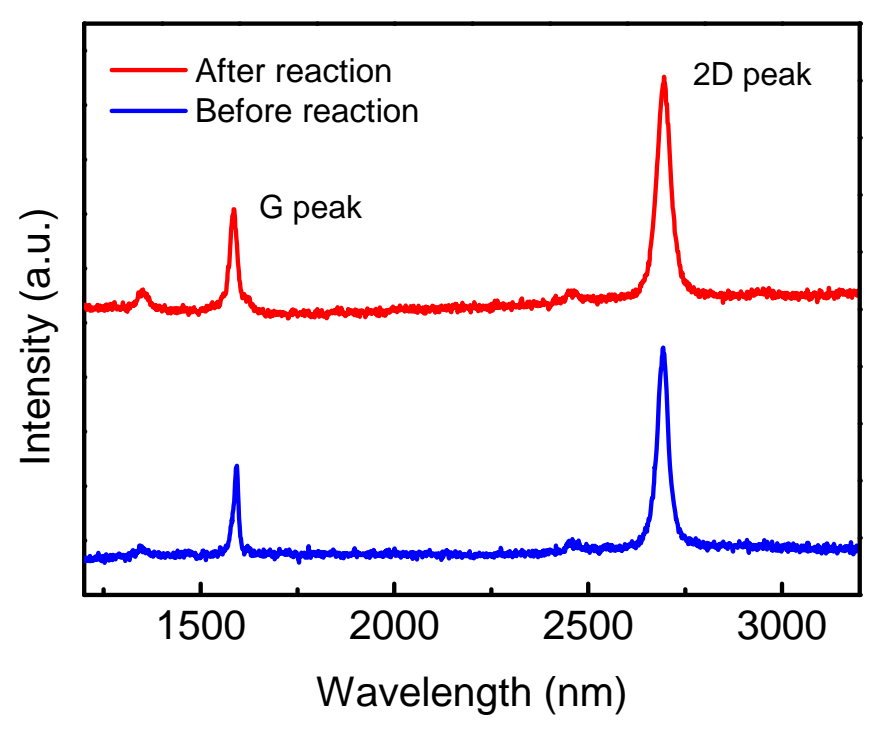

Figure S1. Raman spectrum of a single graphene layer on $\mathrm{TiO}_{2}$ before and after $\mathrm{H}_{2}$ oxidation. The ratio of $\mathrm{I}_{2 \mathrm{D}} / \mathrm{I}_{\mathrm{G}}$ is 2.4 (before reaction), indicating that the graphene layer on $\mathrm{TiO}_{2}$ is a single layer. After the $\mathrm{H}_{2} / \mathrm{O}_{2}$ reaction, $\mathrm{I}_{2 \mathrm{D}} / \mathrm{I}_{\mathrm{G}}$ is 2.3 , indicating that the graphene was not affected by the chemical reaction. 


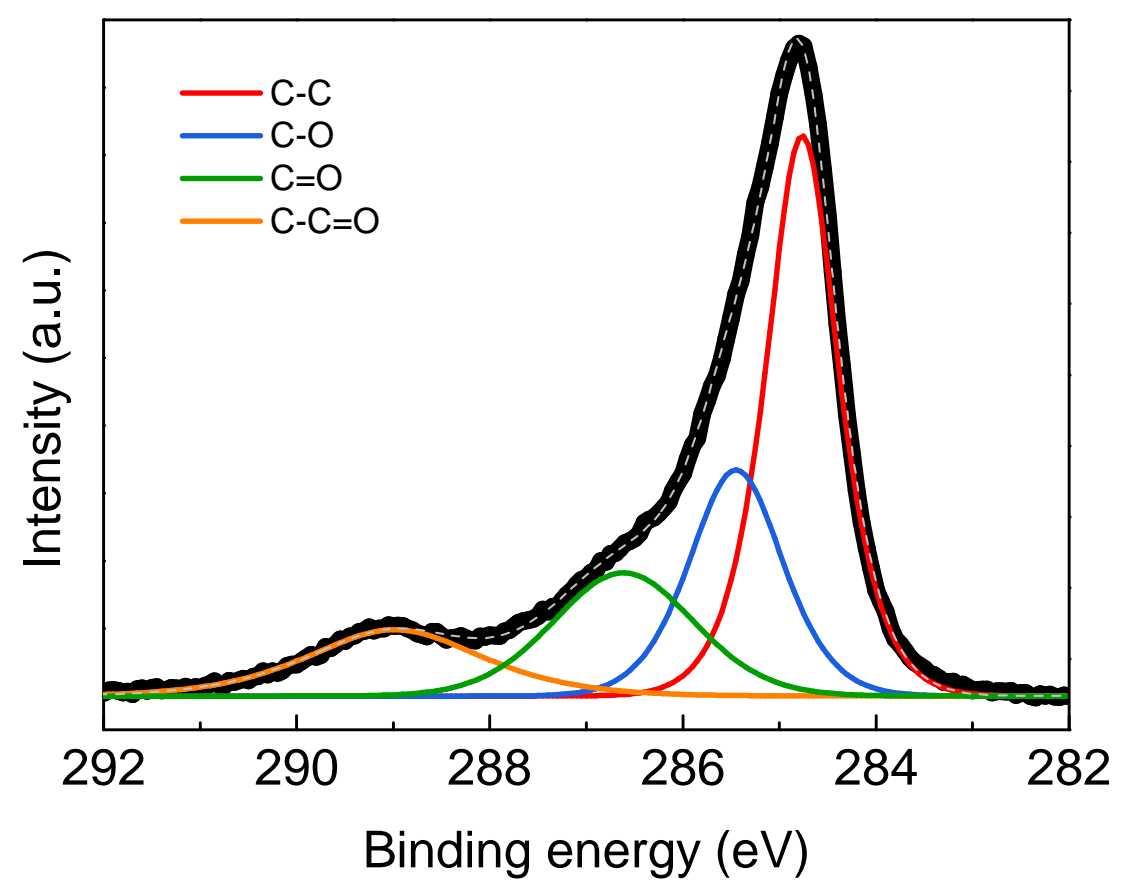

Figure S2. XPS characterization: $\mathrm{C} 1 \mathrm{~s}$ peak of graphene on $\mathrm{TiO}_{2}$.

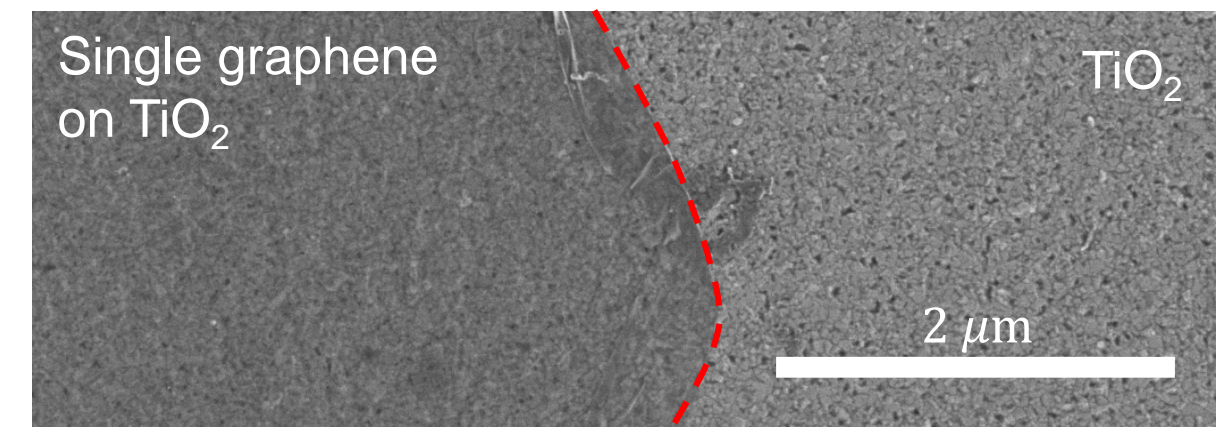

Figure S3. SEM image showing the boundary between the graphene and $\mathrm{TiO}_{2}$ layer before the deposition of Pt NPs. 
- Synthesis of Pt nanoparticles

Pt nanoparticles (NPs) were synthesized by controlling the concentration ratios of Pt(II) to $\mathrm{Pt}(\mathrm{IV})$ in the initial solution because particle size is determined by changing the nucleation rate and the number of nuclei. The synthesis procedure is as follows: First, a total of $20 \mathrm{mg}$ $\left(\mathrm{NH}_{4}\right)_{2} \mathrm{PtCl}_{6}, 120 \mathrm{mg} \mathrm{N}\left(\mathrm{CH}_{3}\right)_{4} \mathrm{Br}^{-}$, and $100 \mathrm{mg}$ PVP (in terms of the repeating unit; $\mathrm{M}_{\mathrm{W}}=$ 29,000 ) were dissolved into $10 \mathrm{~mL}$ ethylene glycol in a $25 \mathrm{~mL}$ round-bottom flask at room temperature. The mixture was heated to $180^{\circ} \mathrm{C}$ in an oil bath for $3 \mathrm{~min}$ and held for $20 \mathrm{~min}$ under argon protection and magnetic stirring. The solution was cooled to room temperature when the color of the solution became dark brown. Finally, the Pt NPs were dispersed in ethanol and washed by repeated centrifugation. The shape and size distribution of the asfabricated $4.5 \mathrm{~nm}$ Pt NPs were studied using transmission electron microscopy (TEM) (see Figures 1c and S4). High-resolution transmission electron microscopy (HR-TEM) was performed using a Tecnai TF30 ST.
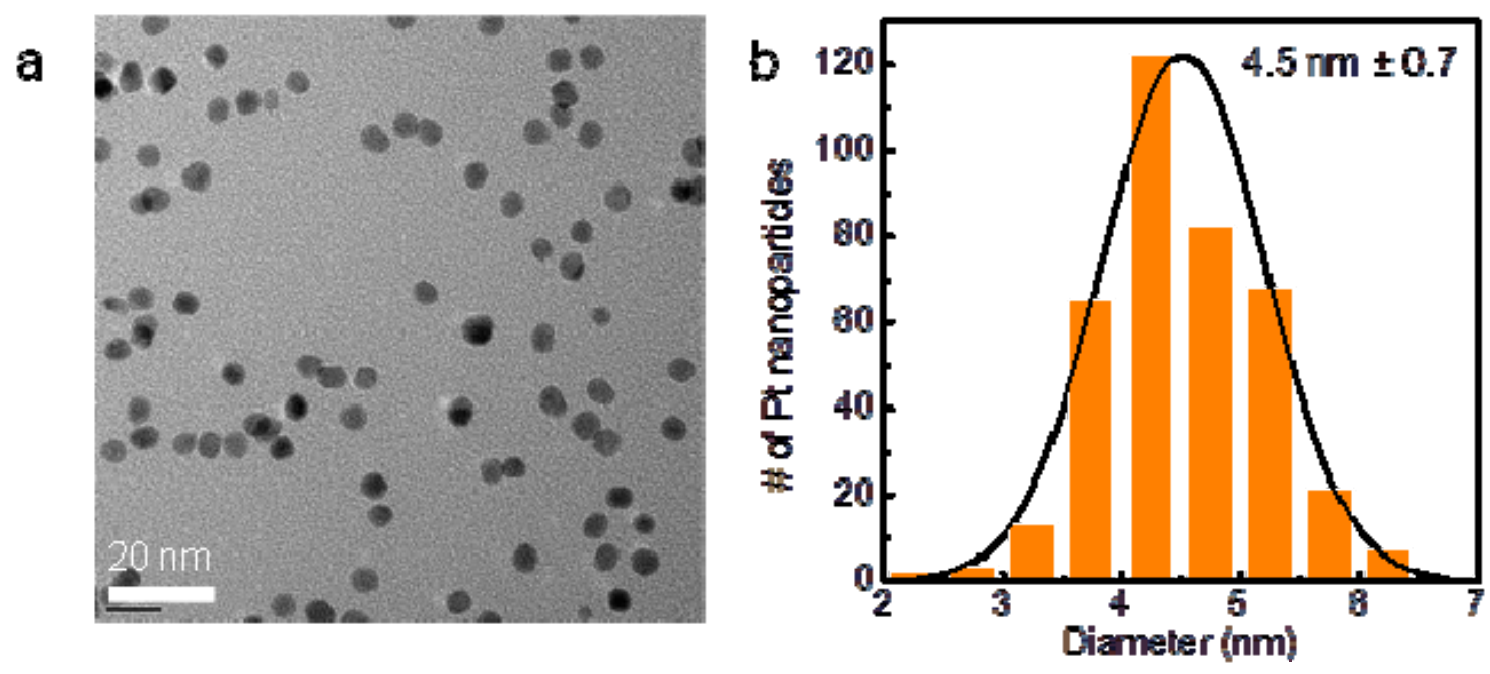

Figure S4. (a) TEM image of $4.5 \mathrm{~nm}$ Pt NPs measured on a copper TEM grid. (b) Size distribution histogram of the $4.5 \mathrm{~nm}$ Pt NPs. 


\section{- Fabrication of Pt NPs/graphene/ $\mathrm{TiO}_{2}$ nanodiode}

First, a $250 \mathrm{~nm}$ Ti layer was deposited using an aluminum shadow mask $\left(4 \times 6 \mathrm{~mm}^{2}\right)$ on a $p$-type $\mathrm{Si}(100)$ wafer covered by $500 \mathrm{~nm} \mathrm{SiO}_{2}$ using electron beam evaporation with a titanium target; it was then annealed in air to transform the Ti layer into an n-type $\mathrm{TiO}_{2}$ film. After that, the electrode - including a $50 \mathrm{~nm}$ Ti layer and a $150 \mathrm{~nm}$ Au layer-was evaporated through a second shadow mask by electron beam evaporation, in which the titanium is the ohmic contact with the $\mathrm{TiO}_{2}$ layer and the Au provides the nanodiode's two ohmic electrodes. Finally, a single graphene $\left(5 \times 5 \mathrm{~mm}^{2}\right)$ layer synthesized using CVD on $\mathrm{Cu}$ foil (Alfa Aesar, $99.9999 \%$ purity) was transferred to the selected area of the device. The graphene used in these experiments was prepared using the following steps: coat with poly(methyl methacrylate) (PMMA), etch the backside of the $\mathrm{Cu}$ foil, and, finally, remove the PMMA placed on the graphene surface.

As-synthesized $4.5 \mathrm{~nm}$ Pt NPs were deposited in 2D arrays on the surface of the graphene $/ \mathrm{TiO}_{2}$ nanodiodes using the Langmuir-Blodgett technique (Nima Technology, M611). We began by dropping the Pt NPs/ethanol solution onto the water subphase in a trough. The water-supported thin-film layer of NPs was then compressed with mobile barriers at a rate of $15 \mathrm{~cm}^{2} / \mathrm{min}$ while the surface pressure was monitored with a Wilhelmy plate. Finally, the submerged graphene/ $\mathrm{TiO}_{2}$ nanodiode - whose non-active part was covered by Teflon tape-was lifted, thus resulting in the formation of a 2D monolayer of Pt NPs on the active surface area of the nanodiodes. 


\section{- Estimation of Schottky barrier height}

According to the thermionic emission theory, the current through a Schottky diode is described by

$$
\begin{aligned}
& I=I_{\text {sat }}\left[\exp \left(\frac{q\left(V-I R_{S}\right)}{\eta k_{B} T}\right)-1\right] \\
& I_{\text {sat }}=A A^{* *} T^{2} \exp \left(-\frac{q \varphi_{b}}{k_{B} T}\right)
\end{aligned}
$$

where $I_{\text {sat }}$ is the reverse saturation current, $q$ is the elementary charge, $\eta$ is the ideality factor, $k_{B}$ is the Boltzmann constant, $\mathrm{T}$ is the temperature, $\mathrm{V}$ is the voltage, $R_{S}$ is the series resistance, $A$ is the diode area, $A^{*}$ is the Richardson constant, and $\varphi_{b}$ is the Schottky barrier height. After taking the logarithm, eq. (1) can be written as

$$
\ln (I)=\ln \left(I_{\mathrm{sat}}\right)+\frac{q\left(V-I R_{S}\right)}{\eta k_{B} T}
$$

Using experimental data from the current-voltage (I-V) curves, we can extract the saturation current $\left(I_{\text {sat }}\right)$ from the y-axis intercept at different temperatures. Subsequently, the Schottky barrier height was estimated from the current data at various temperatures using the following relationship:

$$
\ln \left(\frac{I_{s a t}}{T^{2}}\right)=-\frac{q \varphi_{b}}{k_{B} T}+\ln \left(A A^{* *}\right)
$$

All of the values we calculated are summarized in Table 1 for the Pt NPs/graphene/TiO 2 nanodiode and we confirmed the $0.54 \mathrm{eV}$ Schottky barrier height. Meanwhile, we also estimated that the Schottky barrier height for the graphene/ $\mathrm{TiO}_{2}$ nanodiode without Pt NPs is $0.51 \mathrm{eV}$, which indicates that the Pt NPs on the graphene slightly increase the work function of the graphene (Figure S5). All of the electrical characterization of the nanodiodes was conducted using a Keithley Instrumentation 2400 sourcemeter under various chemical reaction conditions. 


\begin{tabular}{ccccc}
\hline Temperature $\left({ }^{\circ} \mathrm{C}\right)$ & $\boldsymbol{q} / \boldsymbol{k}_{\boldsymbol{B}} \boldsymbol{T}(\mathbf{1} / \boldsymbol{K})$ & $\boldsymbol{l n}\left(\boldsymbol{I}_{\text {sat }}\right)$ & $\boldsymbol{I}_{\text {sat }}(\boldsymbol{n} \boldsymbol{A})$ & $\boldsymbol{\operatorname { l n } ( \boldsymbol { I } _ { \text { sat } } / \boldsymbol { T } ^ { \mathbf { 2 } } )}$ \\
\hline 25 & 38.97 & -17.01 & 40.86 & -28.41 \\
35 & 37.71 & -16.32 & 81.96 & -27.78 \\
45 & 36.52 & -15.59 & 169.6 & -27.11 \\
55 & 35.41 & -14.94 & 326.1 & -26.52 \\
65 & 34.36 & -14.24 & 657.4 & -25.88 \\
75 & 33.37 & -13.69 & 1137 & -25.39 \\
\hline
\end{tabular}

Table 1. Summary of calculated data with the reverse saturation current in the $I-V$ curves for Schottky barrier height estimation for the Pt NPs/graphene/ $\mathrm{TiO}_{2}$ nanodiode.

a

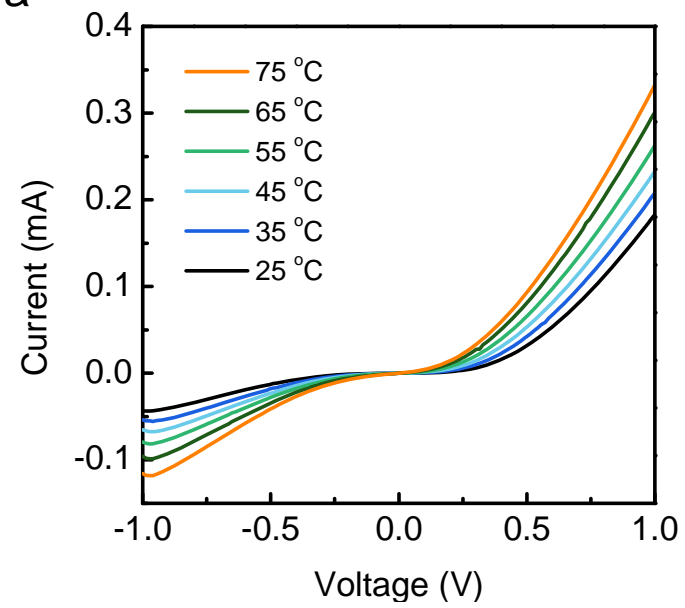

b

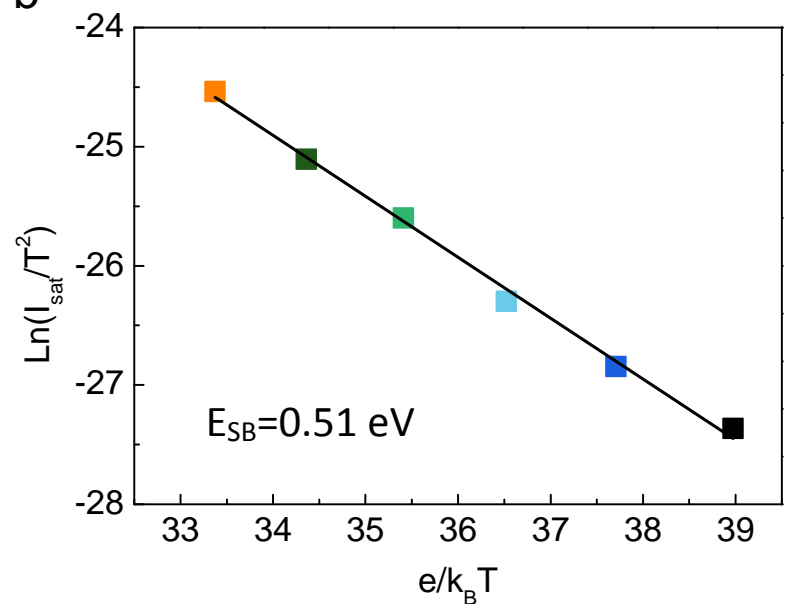


Figure S5. (a) Temperature-dependent current-voltage (I-V) curves of graphene/ $\mathrm{TiO}_{2}$ nanodiodes without Pt NPs. (b) Line fitting for the temperature-dependent saturation current of the device yields a Schottky barrier height of $0.51 \mathrm{eV}$ from the slope of the fitted line.

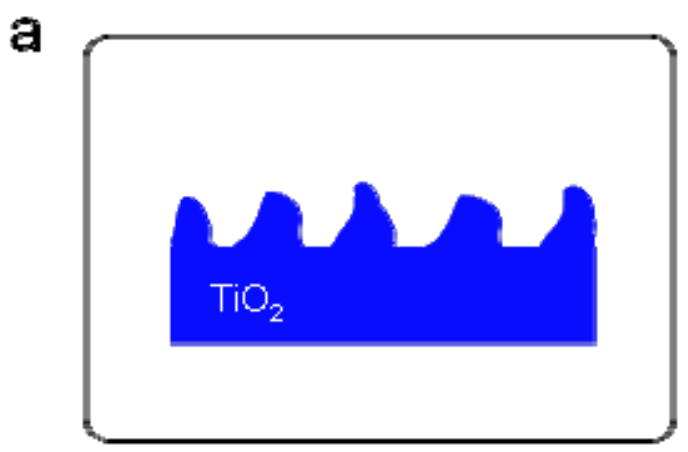

b
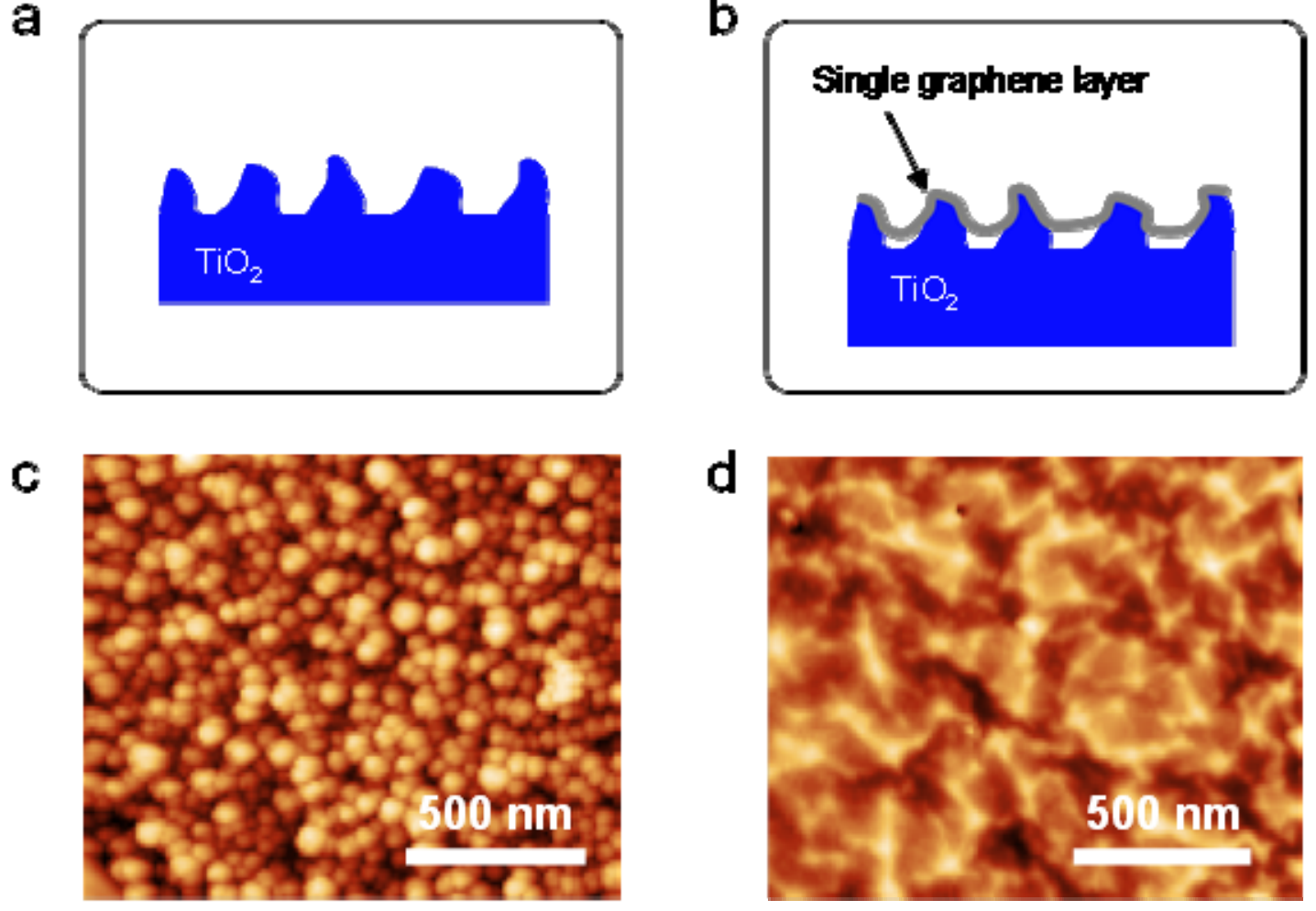

Figure S6. (a) Cross-sectional scheme and (c) atomic force microscopy of the $\mathrm{TiO}_{2}$ film. (b) Cross-sectional scheme and (d) atomic force microscopy of the graphene/ $\mathrm{TiO}_{2}$ nanodiode. The root mean square values of the roughness of the $\mathrm{TiO}_{2}$ film and graphene $/ \mathrm{TiO}_{2}$ nanodiode were found to be 9.5 and $6.2 \mathrm{~nm}$, respectively, which indicate that the actual contact area of graphene $/ \mathrm{TiO}_{2}$ is limited by the roughness of the $\mathrm{TiO}_{2}$ layer, as illustrated in (b). 


\section{- Rate of $\mathrm{H}_{2}$ oxidation}

The $\mathrm{H}_{2}$ oxidation reaction was performed in an ultrahigh-vacuum batch reactor (1L) with a base pressure of $5.0 \times 10^{-8}$ Torr. The reaction chamber was evacuated and isolated with a gate valve before it was charged with a 5, 15, or 25 Torr $\mathrm{H}_{2}$ and $\mathrm{O}_{2}$ mixture at room temperature so that the total pressure in the chamber was equal to atmospheric pressure. The sample consisting of Pt NPs on a $\mathrm{SiO}_{2}$ substrate was placed on a ceramic heater in a batch reactor; the temperature was monitored by a thermocouple. The temperature fluctuations were measured to be less than $0.5 \mathrm{~K}$. The reaction mixture was circulated continuously through the reaction line by a Metal Bellows recirculation pump at a rate of $2 \mathrm{~L} \mathrm{~min}^{-1}$. After equilibrating for $1 \mathrm{~h}$, the reaction mixture was continuously analyzed through an online gas chromatograph. While heating the sample on a heater $\left(80-110^{\circ} \mathrm{C}\right)$, the reaction mixture was separated for analysis using a DS iGC 7200 gas chromatograph equipped with a thermal conductivity detector and a $6 \mathrm{ft}$ long, 1/8" outer diameter stainless steel 80/100 mesh size column. $\mathrm{H}_{2} \mathrm{O}$ conversion was reported in terms of turnover frequency (TOF) and was calculated on the basis of product molecules of $\mathrm{H}_{2} \mathrm{O}$ produced per metal surface site per second of reaction time by monitoring the amount of $\mathrm{H}_{2}$ produced. 


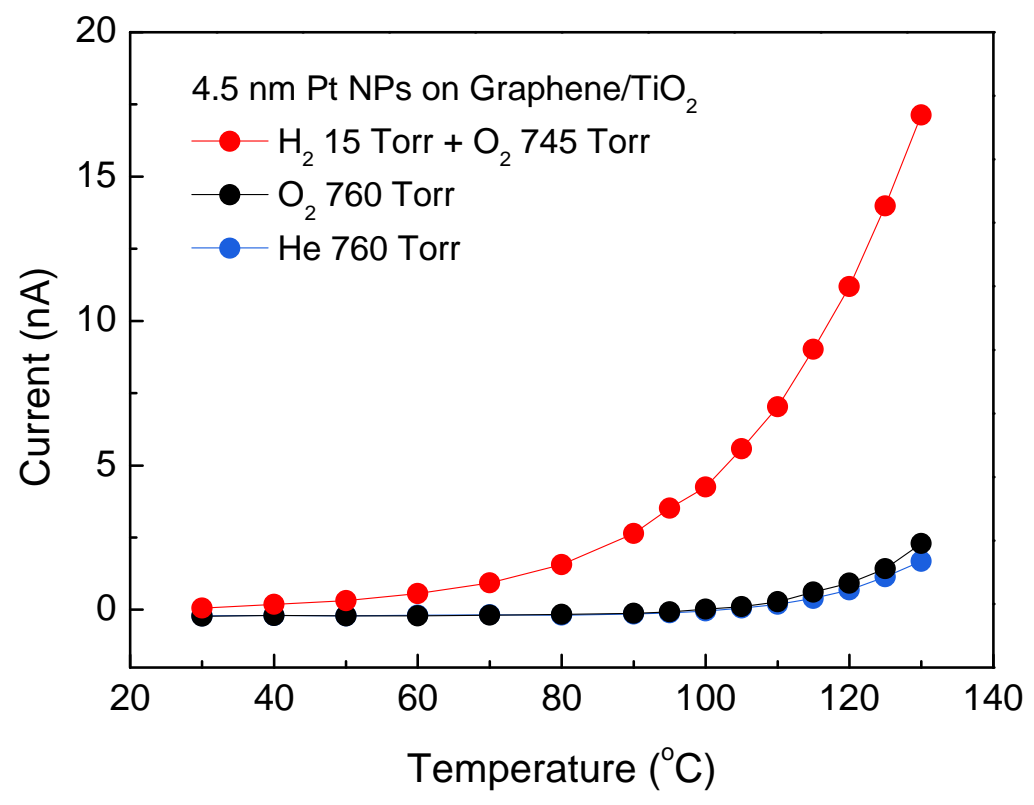

Figure S7. Temperature dependence of the current from the $\mathrm{Pt} \mathrm{NPs} /$ graphene/ $/ \mathrm{TiO}_{2}$ nanodiodes measured in the $\mathrm{H}_{2}+\mathrm{O}_{2}$ gas mixture, in pure $\mathrm{O}_{2}$, and in pure He. The thermoelectric currents from the Pt NPs/graphene/ $/ \mathrm{TiO}_{2}$ nanodiode, measured in both pure $\mathrm{O}_{2}$ and pure $\mathrm{He}$, are nearly identical. 


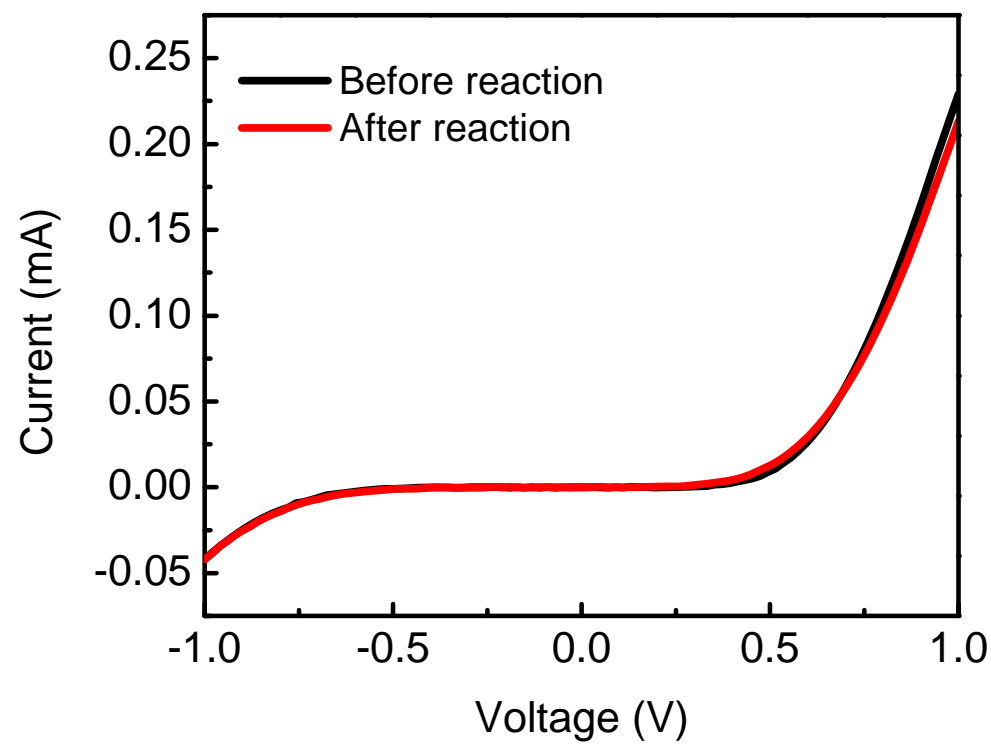

Figure S8. (a) $\mathrm{I}-\mathrm{V}$ curves for the $\mathrm{Pt} \mathrm{NPs} /$ graphene/ $\mathrm{TiO}_{2}$ nanodiodes before and after the chemical reaction was carried out under a mixture of 5 Torr $\mathrm{H}_{2}$ and 755 Torr $\mathrm{O}_{2}$ and at temperatures of $30-130^{\circ} \mathrm{C}$. 

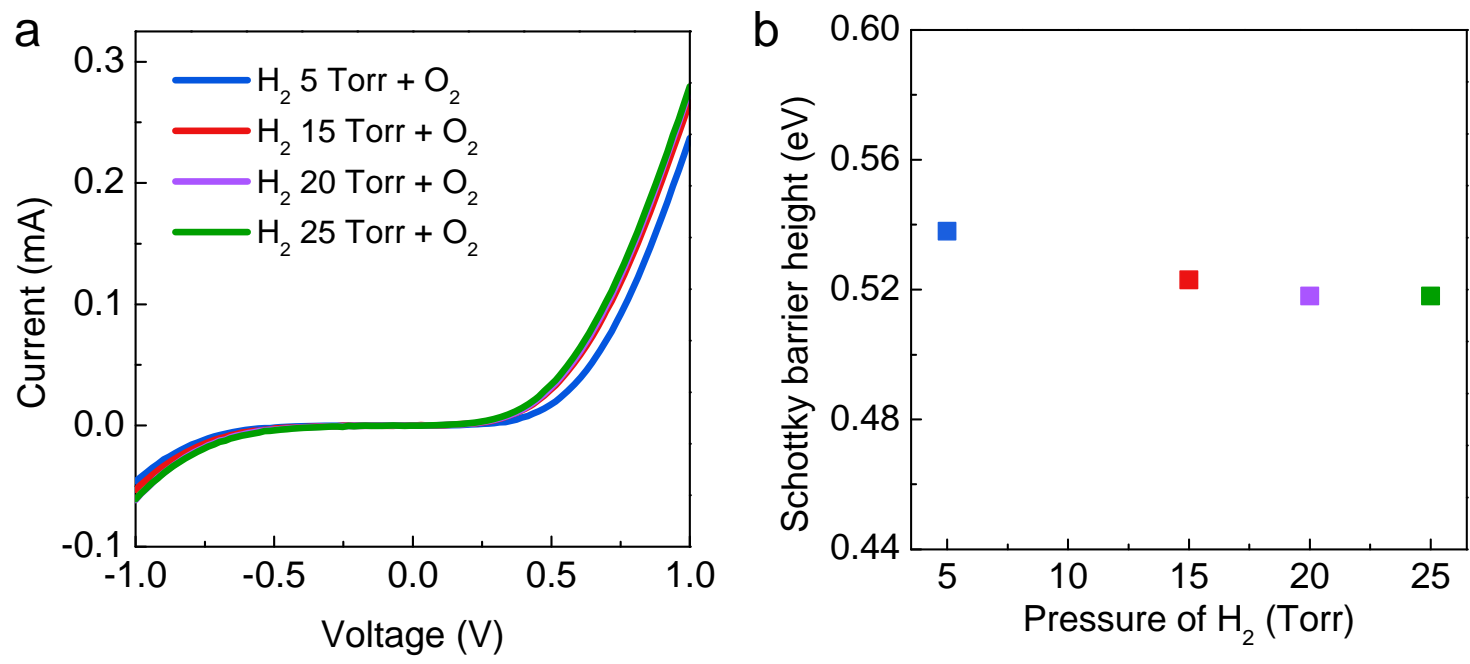

Figure S9. (a) $\mathrm{I}-\mathrm{V}$ curves of the graphene $/ \mathrm{TiO}_{2}$ nanodiodes decorated with $4.5 \mathrm{~nm}$ Pt NPs at different $\mathrm{H}_{2}$ concentrations. (b) Schottky barrier versus $\mathrm{H}_{2}$ concentration. 

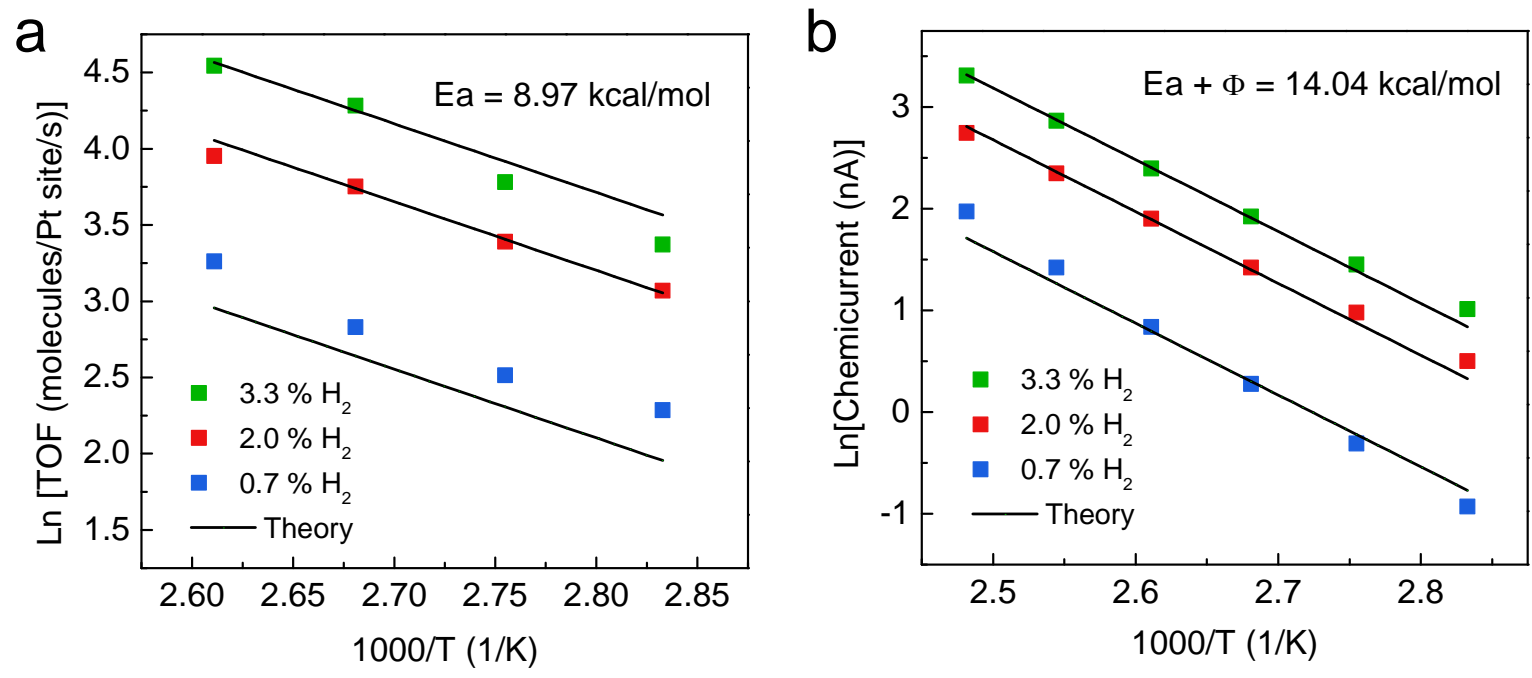

Figure S10. Arrhenius plots obtained from measurement of the (a) chemicurrent and (b) TOF detected by the $\mathrm{Pt} \mathrm{NPs} /$ graphene/ $/ \mathrm{TiO}_{2}$ nanodiodes at various $\mathrm{H}_{2}$ partial pressures. 